\title{
1 Conformable bioelectronic interfaces: mapping the road ahead
}

2 Giuseppe Schiavone, Stéphanie P. Lacour

3 Bertarelli Foundation Chair in Neuroprosthetic Technology, Laboratory for Soft Bioelectronics Interface, Institute of 4 Microengineering, Institute of Bioengineering, Centre for Neuroprosthetics, Ecole Polytechnique Fédérale de

5 Lausanne, 1202 Geneva, Switzerland

6 Overline: $10^{\text {th }}$ ANNIVERSARY FOCUS

7 One-sentence summary: Translating conformable bioelectronic interface research into clinical reality 8 foretells a promising future for an aging society.

Today's technologies hold enormous promise for improving health and well-being. Chronic conditions such as cardiac arrhythmia, deafness, Parkinson's disease, diabetes, or chronic pain, are increasingly assessed and treated with wearable or implantable electronic medical devices. Medical technology is evolving at a rapid pace in response to clinical needs, progress in manufacturing, and research and development conducted within medical device companies. In parallel, bioelectronics research in academic laboratories is fueling innovation in materials, device integration and therapeutic applications to answer the increasing demand for medical devices that can meet the expectations of a growing and aging population, support more personalized health care, and harness large-scale health data. In this Focus article, the seventh in a special series celebrating the 10th anniversary of Science Translational Medicine, we discuss recent progress and ongoing challenges posed by the translation of conformable bioelectronic interfaces.

\section{REDUCED INVASIVENESS AND IMPROVED BIOINTEGRATION}

Within the bioelectronics field, miniaturized devices with high conformability to complex anatomical structures and wireless data transfer capabilities are highly desirable. Although smart (wireless network-connected) medical devices, fueled by high tech companies, have become accepted both by patients and practitioners, their form factor (shape, size, and physical specifications) remains a challenge.

Wearable and implantable devices with better biointegration, bi-directional modalities, and higher spatio-temporal resolution compared to current clinically-approved technology are anticipated to emerge as technology advances. Groundbreaking proof-of-concept work in conformable bioelectronic devices a decade ago triggered exciting opportunities. For example, in 2010, Viventi et al. (1) demonstrated a mechanically flexible 288-channel active silicon-based array adhered to the three-dimensional and moving surface of the porcine heart. This flexible array enabled in vivo mapping of cardiac electrophysiology with unmatched spatio-temporal precision. A few months later, Kim et al. (2) reported an ultra-thin system that conformally laminates onto the surface of skin to enable intimate human-machine interfaces with highperformance electrophysiological monitoring functionalities. 
Since 2010, different materials and technologies have been explored (3-6), yet the general consensus suggests that the next technological breakthroughs will be enabled by the efficient and reliable transfer of microelectronic capabilities onto conformable substrates. Microtechnology offers several advantages. The miniaturization capabilities introduced by lithographic patterning enable fabrication of devices with higher functional density in smaller form factors, therefore reducing the invasiveness of implantation. The batch fabrication techniques of the microelectronics industry permit manufacturing at markedly lower cost compared to today's state-of-the-art electronic medical devices, which are assembled manually by highly skilled personnel. In addition, microelectronic fabrication frameworks offer wellestablished quality control procedures that can accelerate clinical translation.

In wearable applications, the emerging format is a skin-like patch that hosts thin and/or thinned powering, transmission and transducing devices for imperceptible and ubiquitous physiology monitoring (6). Adhesive solutions for wearable interfaces are a major line of on-going research to bond electronic components together, ensure the bioelectronic devices stay in place and for a long period of time. and offer, if needed, reversibility. The benefits of wearable bioelectronics are the support and continuous tracking of pre-defined biomarkers from people, of any age, in non-clinical environments, such as in the home.

In implantable applications, many designs are driven by the optimization of the mechanical signature of the bioelectronic system. Since mechanics have been shown to play a key role in the onset of foreign body reaction (7), research groups worldwide are developing strategies to make bioelectronics more transparent to the host biology. Numerous solutions are being proposed to mitigate the rejection of implanted devices by the body. The most common perspective in the field is the engineering of mechanical compliance within the implanted devices. This can be achieved by using substrate materials of

58 low Young's modulus (4) or bioresorbable matrices (8); designing reduced stiffness (3, 6), small footprint (5), and radically different form factors such as meshes (9); and advancing wireless, untethered interfaces (6). Other exploratory and complementary solutions include locally administering bioactive molecules to reduce inflammation and promote neural growth (10) or carefully selecting materials enveloping the implanted interfaces based on their surface chemistry (11).

\section{TRANSLATING FORM AND FUNCTION}

Conformability in a bioelectronic interface indicates its ability to envelop a surface and maintain functionality under dynamic and multi-axial deformation. This is an essential property for the man-made interface to comply with the convoluted, moving structures that are typical of biology. For example: the skin on the forehead and around the eyes stretches and compresses extensively. The heart beats 60 to 100 times per minute, sustains a total volume variation of about $8 \%$ throughout the cardiac cycle (12), and operates at this capacity for a lifetime. When the heart beats, periodic variations in arterial blood pressure 
currently unmet by rigid bioelectronic interfaces and significant efforts are deployed towards implementing bioelectronic functions within conformable carrier substrates.

In today's research landscape, there is a trade-off between functionality and conformability: Complex, multi-modal, high channel count systems are typically built on rigid to flexible or bendable substrates, whereas only much simpler devices use soft materials such as elastomers or gels. Advancing conformable bioelectronic interfaces requires the successful combination of these two fronts, with technologies enabling complex functionality on ultra-conformable materials. As new materials and engineering strategies are proposed, considerable research efforts are needed to assess their translational potential, recalibrate expectations, and define a sound way forward to clinical use. Table 1 identifies and summarizes critical challenges associated with the development of bioelectronic interfaces, which once tackled will help to convert technological hype into medical hope. Challenges include: Scaling, hermetic encapsulation, system-level integration and compliance to handling in a typical healthcare use scenario. Wireless telemetry and rechargeable batteries are additional components for which there are currently no proscribed paths forward.

Prototypes of implantable bioelectronic interfaces are often tested in small animal models, and dimensional scaling requires more than simply a linear transformation of the interface geometry. Translation necessitates adjusting the overall dimensions to fit larger anatomical structures, but also reevaluating the layout and performance of the scaled devices. For example, considering a bioelectronic interface designed to deliver functional electrical stimulation to tissue, the charge injection capacity of a given electrode coating scales down with increasing electrode geometrical surface area. This implies higher current/voltage may be required to deliver equivalent charges per phase to the tissue, or an improved electrode coating may need to be introduced. Compatibility with medical implantable pulse generators, especially in terms of voltage compliance and leads resistive load, should also be anticipated. Iterative design cycles are therefore needed to scale the electrical and electrochemical performance of the bioelectronic interface.

\section{SURMOUNTING FAILURES AND BYPASSING BOTTLENECKS}

A common failure mechanism of current bioelectronics is the ingress of conductive fluids over time (blood, interstitial fluid, cerebrospinal fluid, but also sweat and water), resulting in loss of insulation between separate conductors. This is particularly challenging for implantable bioelectronics as water permeability of common substrate materials used in conformable bioelectronics (silicones or plastic foils) does not guarantee sufficient insulation over years when implanted in the body. Brittle inorganic layers such as oxides or nitrides offer excellent barrier properties, but at the expense of conformability. A prominent solution that is currently being investigated is the integration of thin multilayer stacks of polymer and inorganic insulators, which promise a combination of conformability and barrier properties. Future embodiments will have to demonstrate reliable material interfaces and integration on soft carrier substrates. 
Another current bottleneck for the successful translation of bioelectronic interfaces is the need for reliable integration in standalone, fully implantable systems. Today, most bioelectronic interfaces of any kind must be physically connected to the corresponding driving electronics that relay electrical signals into and out of the body via transdermal connectors or wireless transmission. Wires and cables prevent truly wearable applications. Although electronic boards can be packaged in hermetic implantable capsules, the challenge lies in the interconnection of high channel count devices to such packages with reliable feedthroughs. Current implantable technology enables implanted systems with this type of connection scheme for pacemakers and neuromodulation therapy devices, which use only a small number of channels $(<16)$. Although the approach originally shown by Viventi et al. (1) reduced 288 channels to a mere 36 multiplexed channels, new feedthrough solutions are required for devices with higher channel counts to be implanted chronically with minimal invasiveness.

Finally, the ability of devices to perform "as expected" and "on demand" by the user (surgeons, clinicians) in the intended setting (inside and outside of the operating theatre or medical unit) is often overlooked in research prototypes. Rigid devices are relatively easy to implant, position, and manipulate during procedures, but do not provide conformal contact with tissue. Conversely, conformable materials require ad-hoc surgical tools that enable the surgeon to easily place the device where needed and remove it when required. With time, researchers may become accustomed to handling conformable materials, however, it is important to consider and include during the initial design, development, and modification stages all of the tools that will be required for ultimate clinical deployment and use of a device or system by healthcare professionals.

\section{REGULATION AND THE ROAD AHEAD}

From a technical point of view, the inherent design freedom and rapid manufacturability offered by conformable microtechnology pose no obstacles to current methods of surgical planning and device use. However, developments in regulatory compliance for medical devices may tend to favor status quo over innovation, imposing ever stricter validation protocols on medical device producers and clinicians willing to introduce bold changes to medical practice. Such divergence between research directions and the norms regulating innovation in the clinic is another aspect of bioelectronic interface development that warrants careful consideration and ongoing dialogue.

The different ethical facets of current and future research in bioelectronics also require further discussion. Academic work seldom crosses laboratory boundaries to venture into the clinic, and leveraging innovative technology to produce new medical devices for healthcare use is a long and costly challenge. From the academic perspective, considerable effort is required to bring new technology to the clinic, with important validation milestones that must be demonstrated before applying for a first clinical trial. Extensive tests must be conducted in compliance with relevant standards or, in the absence thereof, convincing proof must be provided regarding the fitness and robustness of new candidate devices. In vivo testing using translational animal models plays an important role in demonstrating long-term functionality 
146 of new devices when coupled with existing clinical systems and practices. This process entails heavy

147 investment into development work which, per se, is not sufficiently acknowledged and valued as scientific

148 innovation, and is therefore often difficult to publish in the scientific literature.

149 From an industrial perspective, regulatory compliance and a widespread conservative approach in 150 medical practice often means that medical technology companies tend to remain anchored to well151 established frameworks. As extensive deviation from standard practice entails higher approval barriers, 152 the general trend in the field is to carefully weigh innovation against regulatory requirements. This 153 scenario is in net opposition to the perspective ideological trend of personalized healthcare, which 154 advocates that both technology and therapy must be tailored to the individual needs of each patient, with 155 the aim of improving the therapeutic outcome of treatments. Adaptation of policies should be the subject of 156 discussions among all stakeholders, including clinicians and technologists.

157 Over the past decade, innovation in conformable bioelectronics has advanced rapidly to the point 158 that it is inconceivable that conformable interfaces will not eventually convert to a standard in healthcare. 159 Although the translational road is arduous and costly, investigators should be encouraged to push their 160 laboratory research towards clinical adoption. Multidisciplinary collaboration and training programs across 161 the life sciences, engineering, and medicine should be fostered, and long-term funding support through 162 public and private partnerships intensified to maximize the impact of technological research and 163 productively, and to bring new conformable bioelectronic technologies to patient care. 
1. J. Viventi, D.-H. Kim, J. D. Moss, Y.-S. Kim, J. A. Blanco, N. Annetta, A. Hicks, J. Xiao, Y. Huang, D. J. Callans, J. A. Rogers, B. Litt, A Conformal, Bio-Interfaced Class of Silicon Electronics for Mapping Cardiac Electrophysiology, Sci. Transl. Med. 2, 24ra22-24ra22 (2010).

2. D.-H. Kim, N. Lu, R. Ma, Y.-S. Kim, R.-H. Kim, S. Wang, J. Wu, S. M. Won, H. Tao, A. Islam, K. J. Yu, T. Kim, R. Chowdhury, M. Ying, L. Xu, M. Li, H.-J. Chung, H. Keum, M. McCormick, P. Liu, Y.-W. Zhang, F. G. Omenetto, Y. Huang, T. Coleman, J. A. Rogers, Epidermal electronics, Science 333, 838-843 (2011).

3. D. Khodagholy, J. N. Gelinas, T. Thesen, W. Doyle, O. Devinsky, G. G. Malliaras, G. Buzsáki, NeuroGrid: recording action potentials from the surface of the brain, Nat. Neurosci. 18, 310 (2015).

4. I. R. Minev, P. Musienko, A. Hirsch, Q. Barraud, N. Wenger, E. M. Moraud, J. Gandar, M. Capogrosso, T. Milekovic, L. Asboth, R. F. Torres, N. Vachicouras, Q. Liu, N. Pavlova, S. Duis, A. Larmagnac, J. Vörös, S. Micera, Z. Suo, G. Courtine, S. P. Lacour, Electronic dura mater for long-term multimodal neural interfaces, Science 347, 159-163 (2015).

5. S. Guan, J. Wang, X. Gu, Y. Zhao, R. Hou, H. Fan, L. Zou, L. Gao, M. Du, C. Li, Y. Fang, Elastocapillary self-assembled neurotassels for stable neural activity recordings, Sci. Adv. 5, eaav2842 (2019).

6. H. U. Chung, B. H. Kim, J. Y. Lee, J. Lee, Z. Xie, E. M. Ibler, K. Lee, A. Banks, J. Y. Jeong, J. Kim, C. Ogle, D. Grande, Y. Yu, H. Jang, P. Assem, D. Ryu, J. W. Kwak, M. Namkoong, J. B. Park, Y. Lee, D. H. Kim, A. Ryu, J. Jeong, K. You, B. Ji, Z. Liu, Q. Huo, X. Feng, Y. Deng, Y. Xu, K.-I. Jang, J. Kim, Y. Zhang, R. Ghaffari, C. M. Rand, M. Schau, A. Hamvas, D. E. Weese-Mayer, Y. Huang, S. M. Lee, C. H. Lee, N. R. Shanbhag, A. S. Paller, S. Xu, J. A. Rogers, Binodal, wireless epidermal electronic systems with insensor analytics for neonatal intensive care, Science 363, eaau0780 (2019).

7. D. E. Discher, P. Janmey, Y.-L. Wang, Tissue cells feel and respond to the stiffness of their substrate, Science 310, 1139-1143 (2005).

8. D.-H. Kim, J. Viventi, J. J. Amsden, J. Xiao, L. Vigeland, Y.-S. Kim, J. A. Blanco, B. Panilaitis, E. S. Frechette, D. Contreras, D. L. Kaplan, F. G. Omenetto, Y. Huang, K.-C. Hwang, M. R. Zakin, B. Litt, J. A. Rogers, Dissolvable films of silk fibroin for ultrathin conformable biointegrated electronics, Nat. Mater. 9, 511-517 (2010).

9. X. Yang, T. Zhou, T. J. Zwang, G. Hong, Y. Zhao, R. D. Viveros, T.-M. Fu, T. Gao, C. M. Lieber, Bioinspired neuron-like electronics, Nat. Mater. 18, 510 (2019).

10. T. D. Y. Kozai, A. S. Jaquins-Gerstl, A. L. Vazquez, A. C. Michael, X. T. Cui, Dexamethasone retrodialysis attenuates microglial response to implanted probes in vivo, Biomaterials 87, 157-169 (2016).

11. N. de la Oliva, M. Mueller, T. Stieglitz, X. Navarro, J. del Valle, On the use of Parylene C polymer as substrate for peripheral nerve electrodes, Sci. Rep. 8, 5965 (2018).

12. M. Carlsson, P. Cain, C. Holmqvist, F. Stahlberg, S. Lundback, H. Arheden, Total heart volume variation throughout the cardiac cycle in humans, Am. J. Physiol.-Heart Circ. Physiol. 287, H243-H250 (2004).

(


Competing interests:

210

211

The authors have no competing interests.

212

213

Acknowledgments:

216

The authors would like to acknowledge financial support by the Bertarelli Foundation and the European Union's Horizon 2020 research and innovation programme under the Marie Skłodowska-Curie grant

217 agreement No. 665667.

218 
220 Table 1 - Innovative strategies address challenges in developing next-generation conformable and

221 implantable bioelectronic interfaces.

\begin{tabular}{|c|c|c|c|}
\hline $\begin{array}{l}\text { Conformable } \\
\text { microfabrication }\end{array}$ & $\begin{array}{l}\text { Hermetic } \\
\text { encapsulation }\end{array}$ & $\begin{array}{l}\text { System-level } \\
\text { integration }\end{array}$ & Regulatory adaptation \\
\hline $\begin{array}{l}\text { Manufacturing } \\
\text { standards for thin-film } \\
\text { devices on conformable } \\
\text { carrier substrates }\end{array}$ & $\begin{array}{l}\text { Multilayered stacks of } \\
\text { polymer/inorganic } \\
\text { barriers }\end{array}$ & $\begin{array}{l}\text { Compact wireless } \\
\text { transmission modules }\end{array}$ & $\begin{array}{l}\text { Policy changes for } \\
\text { approval of tailor-made } \\
\text { medical devices }\end{array}$ \\
\hline $\begin{array}{l}\text { Hybrid integration of } \\
\text { rigid (CMOS)* } \\
\text { components and } \\
\text { polymer-based } \\
\text { transducers }\end{array}$ & $\begin{array}{l}\text { Deposition processes, } \\
\text { interface and barrier } \\
\text { properties of inorganic } \\
\text { films such as silicon } \\
\text { carbide ( } \mathrm{SiC} \text { ) and } \\
\text { hafnium oxide }\left(\mathrm{HfO}_{2}\right)\end{array}$ & $\begin{array}{l}\text { Power management } \\
\text { (new battery } \\
\text { technology, battery life / } \\
\text { heating) }\end{array}$ & $\begin{array}{l}\text { New mechanical norms } \\
\text { for conformable devices }\end{array}$ \\
\hline $\begin{array}{l}\text { Fabrication of soft } \\
\text { active }^{\star \star} \text { components }\end{array}$ & $\begin{array}{l}\text { Implantable plug-and- } \\
\text { play connectors / } \\
\text { feedthroughs }\end{array}$ & $\begin{array}{l}\text { Complete kits including } \\
\text { tools for clinical use }\end{array}$ & \\
\hline
\end{tabular}

* Complementary metal-oxide-semiconductor 